\title{
Checkpoint inhibition of PD-L1 and CTLA-4 in a child with refractory acute leukemia
}

\author{
Larisa Broglie ${ }^{1,2}$, Jill Gershan ${ }^{1} \&$ Michael J Burke ${ }^{*, 1}$ \\ ${ }^{1}$ Department of Pediatrics, Division of Hematology/Oncology \& Blood \& Marrow Transplantation, Medical College of Wisconsin, \\ Milwaukee, WI, 53226, USA \\ ${ }^{2}$ Department of Pediatric, Division of Hematology/Oncology \& Blood \& Marrow Transplantation, Columbia University Medical \\ Center, New York, NY, 10027, USA \\ *Author for correspondence: Tel.: +1 414955 4170; Fax: +1 414955 6543; mmburke@mcw.edu
}

Practice points

- Relapsed mixed lineage leukemia can be difficult to treat with standard therapies.

- Checkpoint blockade with nivolumab and ipilimumab has shown promise in treatment of lymphoma and melanoma with growing evidence in other malignancies, such as acute myeloid leukemia (AML).

- Limited data on the efficacy and tolerability of these agents has been reported in pediatrics, although current studies are ongoing.

- We report a patient who tolerated combined therapy with 5-azacitidine, nivolumab and later ipilimumab. The patient was able to focus on her quality of life at home with her family during the 6 weeks of epigenetic and checkpoint inhibitor therapy. However, the patient ultimately died from disease progression.

- Cytokine evaluation after 5-azacytidine and nivolumab therapy may help identify response to therapy. In this patient, the mixed cytokine response may reflect both activated innate immunity against the AML as well as ongoing AML proliferation.

- Further studies are needed to evaluate the efficacy of checkpoint blockade in pediatric AML. Further studies are also needed to determine if cytokine assessment can help determine early response to these therapies.

Childrenwith multiple relapsed or refractory leukemia have dismal survival. Research has identified engagement of immune checkpoint receptors (e.g., PD-1, PD-L1 and CTLA-4) as a mechanism for treatment resistance. For adult cancer, inhibitors of PD-1 (nivolumab) and CTLA-4 (ipilimumab) have shown promise with response rates ranging from 7 to $40 \%$. In vitro studies using acute myeloid leukemia cell lines have shown that acute myeloid leukemia blasts may similarly utilize the PD-1/PD-L1 axis to evade an anticancer immune response. We report the first case of a pediatric patient with multiple relapsed/refractory leukemia treated with nivolumab, ipilimumab and 5-azacytidine who tolerated therapy with brief improvement of symptoms.

First draft submitted: 17 September 2018; Accepted for publication: 11 December 2018; Published online: 16 January 2019

\section{Keywords: acute leukemia $\bullet$ azacytidine $\bullet$ checkpoint inhibitor $\bullet$ epigenetic $\bullet$ ipilimumab $\bullet$ nivolumab $\bullet$ refractory} - relapse

Outcomes for children with leukemia have improved; however, patients with multiple relapsed or refractory disease continue to have dismal survival $[1,2]$. In recent years, research has identified engagement of immune checkpoint receptors as a mechanism of tumor evasion [3]. T-cell checkpoint receptors such as CTLA-4 and PD-1 relay inhibitory signals that modulate T-cell activation. Blocking signaling through checkpoint receptors results in increased T-cell activation, with effector T-cell proliferation and increased cytotoxicity toward cancer cells [4]. Inhibitors of PD1 (nivolumab) and CTLA-4 (ipilimumab) have shown promise for the treatment of advanced melanoma and relapsed Hodgkin's lymphoma with response rates ranging from 7 to $40 \%$ [4-6]. In vitro studies have shown that acute myeloid leukemia (AML) may utilize the PD-1/PD-L1 axis to evade an anticancer immune response [7-10]. These findings have prompted development of clinical trials utilizing checkpoint inhibitors for adult malignancies. However, established efficacy in pediatric hematological malignancies remains limited. 
Here, we report a case of a pediatric patient with multiple relapsed and refractory acute leukemia who tolerated palliative treatment with 5-azacytidine and two checkpoint inhibitors, nivolumab and ipilimumab, allowing her and her family to focus on her quality of life.

\section{Case presentation}

A 4-year-old female presented with persistent fevers and a superficial thigh abscess, unresponsive to outpatient antibiotic treatment. Evaluation revealed an elevated white blood cell count of 84,000 with $74 \%$ peripheral blasts and a mediastinal mass. Flow cytometric analysis reported a single blast immunophenotype $\left(\mathrm{CD} 34^{+}, \mathrm{CD} 117^{+}\right.$,

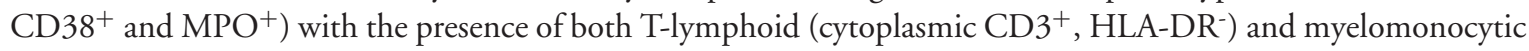
$\left(\mathrm{CD}^{+}, \mathrm{CD} 6^{+}, \mathrm{CD}_{3} 3^{+}\right.$and membrane $\left.\mathrm{CD} 3^{-}\right)$cell markers consistent with mixed phenotype acute leukemia. Based on this phenotype, T-cell acute lymphoblastic leukemia therapy was initially given according to the Children's Oncology Group (COG) protocol AALL0434, but, after 2 days, therapy changed to an AML-directed induction due to concern over a rising white blood cell count of $>100,000$ (Table 1). At the end of induction with cytarabine, daunorubicin and etoposide, a bone marrow evaluation showed complete remission $(<5 \%$ blasts $)$ with minimal residual disease $(\mathrm{MRD})$ present (flow cytometry MRD $=0.5 \%$ ). The patient was switched back to T-ALL therapy to complete a second induction phase (according to COG AALL0434) followed by AALL0434 consolidation with nelarabine and interim maintenance (high-dose methotrexate); however, her MRD persisted, prompting further intensification with cyclophosphamide, etoposide and nelarabine (Table 1). The decision was ultimately made to proceed to allogeneic hematopoietic cell transplantation (HCT), given the inability in achieving an MRD-negative remission (pre-HCT MRD was $0.007 \%$ ). The patient proceeded to a myeloablative umbilical cord blood HCT with total body irradiation $(13.2 \mathrm{~Gy})$, fludarabine $\left(75 \mathrm{mg} / \mathrm{m}^{2}\right)$ and cyclophosphamide $(120 \mathrm{mg} / \mathrm{kg})$. Her posttransplant course was complicated by steroid-responsive grade IV gastrointestinal acute graft-versus-host disease, idiopathic pneumonitis and chronic graft-versus-host disease serositis.

In 320 days post-HCT, the patient developed an isolated submental nodule that was diagnosed histologically as leukemia cutis (expressing the same myeloid phenotype without $\mathrm{CD} 3$ expression from her original diagnosis). Bone marrow aspirate, spinal tap and CT evaluations showed no additional evidence of leukemia. The isolated relapse was completely resected and her parents chose close monitoring without further treatment, instead of reinduction chemotherapy, which was the recommendation of the oncology team. However, over the next few months additional subcutaneous nodules were identified. A PET/CT scan revealed disseminated chloromas. Given this relapse and the known difficulty of cure with traditional chemotherapy, next-generation-sequencing was performed on her original diagnostic bone marrow to identify any potentially targetable mutations. A lone NOTCH mutation was the only lesion identified with a potential therapeutic target. At the time of this relapse, there were no pediatric early phase clinical trials available for her to enroll in. Thus, the patient received multiple reinduction attempts with a variety of chemotherapy salvage combinations, but remission could not be achieved (Table 1).

Given the refractory nature of her disease, which at this time remained as AML without any T-ALL features, and the parent's strong interest in pursuing some form of immunotherapy, we discussed the option of combining checkpoint inhibition (nivolumab) with a DNA methyltransferase inhibitor (5-azacytidine) as was recently reported in adults with refractory AML [11]. After discussion of the potential risks and benefits of this proposed treatment, the family consented to this experimental therapy recommendation as palliative therapy, outside of the context of a clinical trial. Additionally, the parents consented to further research testing performed on their daughter's peripheral blood and bone marrow samples collected during this experimental treatment.

The patient was experiencing significant bone pain at the time prior to starting this palliative therapy for which required hospitalization for aggressive pain management with intravenous narcotics, oral methadone, gabapentin and anxiolytics (lorazepam). The patient thus received her first course of 5 -azacytidine $\left(75 \mathrm{mg} / \mathrm{m}^{2}\right.$ iv. daily, days $1-5)$ and nivolumab (3 mg/kg iv. on days 1 and 14) while in the hospital and tolerated it well without any adverse event (AE). While receiving the combination of 5-azacytidine and nivolumab, her bone pain significantly improved to the point that she was able to be discharged home 5 days later (completing her 5 days of 5 -azacytidine) on oral dilaudid, methadone and gabapentin. The patient remained home with her family, coming to the oncology clinic for twice weekly visits, including for her second dose of nivolumab on day 14 of this treatment cycle, and her pain remained well controlled with oral medications alone. At the start of the 5-azacytidine/nivolumab therapy, the patient's peripheral blood had $1 \%$ blasts present which slowly increased to $10 \%$ by day 14 . Unfortunately, she continued to have persistent and rising disease as her peripheral blood blast percentage increased to $34 \%$ at day 28 of treatment. 
Table 1. Chemotherapy course, complications, and disease response after each treatment.

\begin{tabular}{|c|c|c|}
\hline Therapy & Complications & Response \\
\hline \multicolumn{3}{|l|}{ Diagnosis } \\
\hline $\begin{array}{l}\text { Induction per COG AALL0434 for T-ALL } \\
\text {-Dexamethasone, Vincristine, PEG-asparaginase and } \\
\text { Daunorubicin }\end{array}$ & Rising WBC $>100,000$ & Switched to AAML1031 therapy on Day $\times 2$ \\
\hline $\begin{array}{l}\text { Induction I per AAML1031 } \\
\text {-Etoposide, Daunorubicin, Cytarabine }\end{array}$ & & End of Induction I MRD $0.5 \%$. \\
\hline $\begin{array}{l}\text { Induction following AALL0434 } \\
\text {-Dexamethasone, Vincristine, and Daunorubicin, PEG- } \\
\text { asparaginase }\end{array}$ & Anaphylaxis to peg-asparaginase and Erwinia & End of Induction MRD $0.065 \%$ \\
\hline $\begin{array}{l}\text { Consolidation following AALL0434 } \\
\text {-Nelarabine, Cyclophosphamide, Cytarabine, steroids }\end{array}$ & & End of Consolidation MRD $0.025 \%$ \\
\hline $\begin{array}{l}\text { Interim Maintenance } 1 \\
\text {-High-dose Methotrexate, Vincristine and } \\
6 \text {-mercaptopurine ( } 3 \text { of } 5 \text { doses of Methotrexate } \\
\text { received) }\end{array}$ & & End of Interim Maintenance 1 MRD $0.03 \%$ \\
\hline -Cyclophosphamide, Etoposide, Nelarabine & & End of Cycle MRD $0.025 \%$. \\
\hline Hematopoietic stem cell transplant & & $\begin{array}{l}\text { Pre-HCT MRD } \\
0.007 \%\end{array}$ \\
\hline $\begin{array}{l}\text { Umbilical Cord Blood HCT } \\
\text { Myeloablative conditioning: } \\
\text {-TBI (13.2Gy), Fludarabine }(75 \mathrm{mg} / \mathrm{m} 2) \text {, and } \\
\text { Cyclophosphamide }(120 \mathrm{mg} / \mathrm{kg})\end{array}$ & $\begin{array}{l}\text { Grade IV gut aGVHD } \\
\text { CMV reactivation, BK viuria and viremia, Seizures, } \\
\text { transplant associated microangiopathy, Pneumonitis, } \\
\text { cGVHD (serositis with pleural pericardial effusions) }\end{array}$ & MRD $<0.001 \%$ \\
\hline \multicolumn{3}{|c|}{ Relapse: extramedullary disease and later bone marrow and CNS relapse } \\
\hline -Cyclophosphamide, Etoposide, and Bortezomib & & End of Cycle MRD $0.17 \%$ \\
\hline $\begin{array}{l}\text {-Nelarabine, skin radiation (scalp - } 26 \mathrm{~Gy} \text {, perineum - } \\
21 \mathrm{~Gy} \text {, total skin - 14Gy) with steroids }\end{array}$ & Disease progression & $\begin{array}{l}28 \% \text { blasts, } \\
\text { CNS blasts present } \\
\text { PET - Many diffuse lesions }\end{array}$ \\
\hline $\begin{array}{l}\text {-Gemtuzumab, Low-dose Cytarabine, and intrathecal } \\
\text { Cytarabine }\end{array}$ & $\begin{array}{l}\text { Radiation recall with burns in her perineum } \\
\text { Seizure }\end{array}$ & $\begin{array}{l}\text { End of Cycle MRD } 0.09 \% \text {, CNS negative PET single lung } \\
\text { nodule }\end{array}$ \\
\hline -Venetoclax (120 mg daily for 28 -day cycle) & $\begin{array}{l}\text { ARF from bilateral ureteral obstruction requiring } \\
\text { ureteral stents }\end{array}$ & $\begin{array}{l}\text { End of Cycle } \\
7 \% \text { blasts, } \\
\text { CNS positive } \\
\text { PET lesions in mediastinum, and pelvis }\end{array}$ \\
\hline -Mitoxantrone, High-dose Cytarabine, Gemtuzumab & $\begin{array}{l}\text { Pseudomonas Infections } \\
\text { Cheek cellulitis } \\
\text { Gastritis } \\
\text { Prolonged Count Recovery }\end{array}$ & $\begin{array}{l}\text { End of Cycle } 19 \% \text { blasts } \\
\text { PET negative } \\
\text { Peripheral blood blasts present }\end{array}$ \\
\hline $\begin{array}{l}\text {-Nivolumab ( } 3 \mathrm{mg} / \mathrm{kg} \text { iv. on days } 1 \text { and } 14) \text {, } \\
\text {-Azacytidine }\left(75 \mathrm{mg} / \mathrm{m}^{2} \text { iv. daily } \times 5 \text { days) }\right.\end{array}$ & $\begin{array}{l}\text { Staph epi sepsis } \\
\text { HSV reactivation } \\
\text { Left leg pain secondary to marrow infiltration }\end{array}$ & End of Cycle Persistent disease \\
\hline $\begin{array}{l}\text {-Nivolumab ( } 3 \mathrm{mg} / \mathrm{kg} \text { iv. on day } 1) \\
\text {-Azacytidine }\left(75 \mathrm{mg} / \mathrm{m}^{2} \text { iv. daily } x 5 \text { days) }\right. \\
\text {-Ipilimumab ( } 1 \mathrm{mg} / \mathrm{kg} \text { iv. on day } 3 \text { ) }\end{array}$ & Pseudomonas sepsis & Progressive disease \\
\hline $\begin{array}{l}\text { ARF: Acute renal failure; CMV: Cytomegalovirus; CNS } \\
\text { transplantation; MRD: Minimal residue disease; PET: }\end{array}$ & al nervous system; COG: Children's Oncology Grou & HD: Graft-versus-host disease; HCT: Hemato \\
\hline
\end{tabular}

To assess for any response to checkpoint inhibitor therapy, serum obtained 28 days after starting 5azacytidine/nivolumab was analyzed using the human cytokine/chemokine 65-plex array (Eve Technologies, AB, Canada) that included analysis of the following cytokines/chemokines: CXCL1, CCL1, IFN $\alpha 2$, IFN- $\gamma$, IL-1 $\alpha$, IL-1 $\beta$, IL-1 $\mathrm{r} \alpha$, IL-2, IL-3, IL-4, IL-5, IL-6, IL-7, IL-8, IL-9, IL-10, IL-12 (p40), IL-12 (p70), IL-13, IL-15, IL-16, IL-17A, IL-18, IL-20, IL-21, IL-23, IL-28a, IL-33, IP-10, LIF, MCP-1, MCP-2, MCP-3, MCP-4, MDC, MIP-1 $\alpha$, MIP-1 $\beta$, MIP-1 $\delta$, PDGF-AA, PDGF-AB/BB, RANTES, SDF- $1 \alpha+\beta$, sCD40L, SCF, TARC, TGF $\alpha$, TNF $\alpha$, TNF $\beta$, TPO, TRAIL, TSLP and VEGF. The patient's serum sample was compared with normal adult controls, as no pediatric controls were available. We identified a significant increase (as determined by two-way ANOVA) in the concentration of IL-18, FLT3L, G-CSF, CXCL10 and CCL24 in the patient's serum (Figure 1). While not significant, there was also an elevation in proinflammatory cytokines, IL-6 and TNF-a. Of note, a bone marrow sample was not available for immune phenotyping or assessment of PD-1 or CTLA-4 secondary to poor sample quality. 


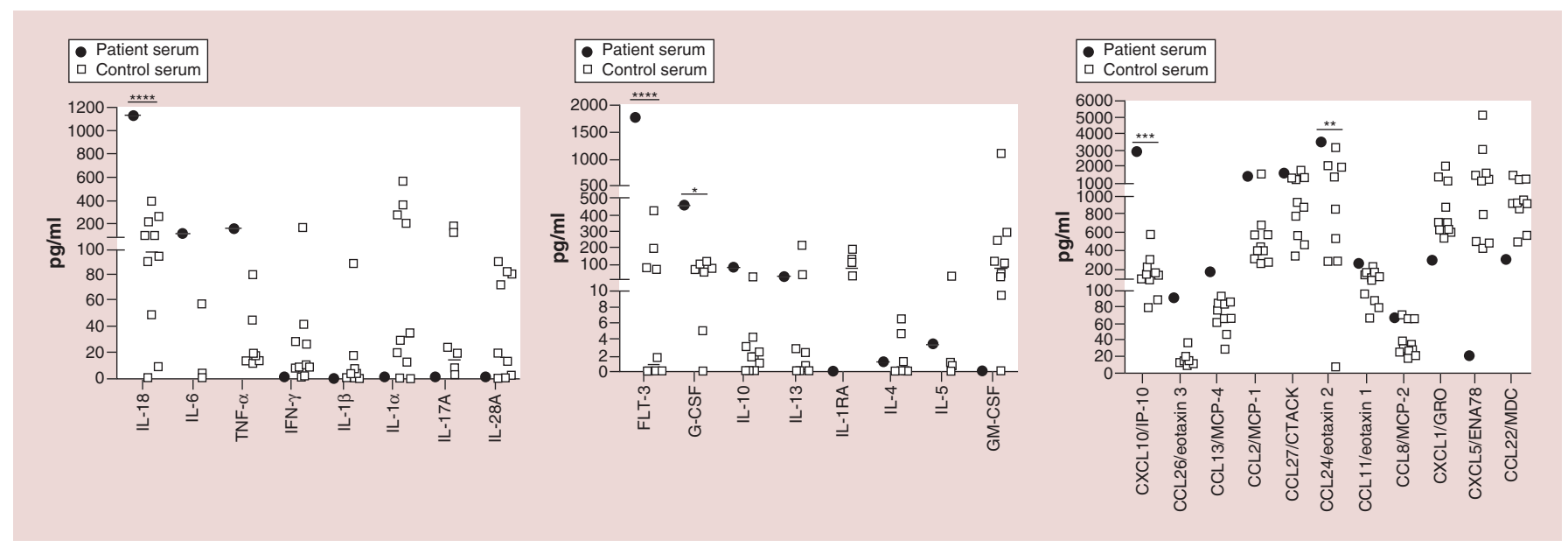

Figure 1. Post-treatment serum cytokines. Serum was obtained after 28 days of treatment with 5-azacytidine and nivolumab. Cytokine concentrations were analyzed by Eve Technologies ( $A B$, Canada) using a Luminex-based platform. Control serum was obtained from normal adult donors.

$* * * * p<0.0001 ; * * * p<0.001 ; * * p<0.01$ and $* p<0.05$

Despite maintaining good pain control and quality of life, given the patient's persistent disease and the family's desire to continue palliative therapy, a second cycle of 5-azacytidine $\left(75 \mathrm{mg} / \mathrm{m}^{2}\right.$ iv. daily, days $\left.1-5\right)$ and nivolumab $(3 \mathrm{mg} / \mathrm{kg}$ iv. on day 1) was started with the addition of ipilimumab $(1 \mathrm{mg} / \mathrm{kg}$ iv. on day 3$)$ in hopes of improving the disease response. The combination of nivolumab and ipilimumab was according to the COG Phase I study (ADVL1412, NCT02304458) which reported safety and tolerability combining the CTLA-4 and PD-1 inhibitor (Table 1). The patient tolerated the addition of the CTLA-4 inhibitor (ipilimumab) to 5-azacytidine and nivolumab well without any AEs, including no reports of further bone pain while continuing her oral pain medications (twice daily methadone, gabapentin three-times a day and Dilaudid as needed). During the two cycles of epigenetic/checkpoint inhibitor therapy, she did not have any elevation of liver transaminases (ALT: 28-47 iU/l; AST: $16-40 \mathrm{iU} / \mathrm{l}$ ) nor significant anemia (range: $7.6-12.0 \mathrm{~g} / \mathrm{dl}$ ) requiring red blood cell transfusions but did require a single platelet transfusion for a platelet count of $10 \times 10^{9}$ per liter (platelet range, $10-110 \times 10^{9} / \mathrm{l}$ ). However, 45 days after starting the first cycle of 5-azacytidine and nivolumab and 11 days after receiving ipilimumab, the patient developed an overwhelming pseudomonas aeruginosa sepsis in the setting of increasing leukemia burden (>65\% peripheral blasts; Figure 2) and succumbed to her leukemia.

\section{Discussion}

We report a pediatric patient with relapsed and refractory mixed phenotype acute leukemia treated with a DNA methyltransferase inhibitor (5-azacytidine) and checkpoint inhibitors (nivolumab and ipilimumab). The patient tolerated the palliative regimen very well for 6 weeks while maintaining a reasonably good quality of life, remaining outside of the hospital and with improved pain control. Importantly, there were no AEs attributed to the epigenetic/checkpoint inhibitor therapy. Unfortunately, our patient continued with persistent leukemia despite this regimen. In an attempt to evaluate the response to checkpoint blockade, we analyzed serum cytokine expression, as we were unable to evaluate PD-1 expression on either peripheral blood or bone marrow blasts.

Studies have shown that checkpoint blockade is associated with a proinflammatory immune response with an increased expression of TNF- $\alpha$, IFN- $\gamma$, IL-2, IL- 6 and IL-17 and can precipitate autoimmunity [12,13]. After 28 days of treatment with 5-azacytidine and nivolumab, our patient had elevated concentrations of proinflammatory cytokines IL-18, CXCL10, FLT3L and CCL24 compared with normal adult control samples. IL-18 is produced upon activation of intracellular innate immune sensors and is considered tumor-suppressive [14]. CXCL10 is released by human AML cells and promotes T-cell chemotaxis [15]. Recombinant CXCL10 has also been shown to inhibit the proliferation of AML precursors [16]. Both IL-18 and CXCL10, induced by checkpoint blockade, suggest an ongoing anti-AML immune response. Unfortunately, our patient also showed evidence of elevated FLT3L and CCL24. FLT3L acts as a mitogenic growth factor for AML cells [17]. CCL24 regulates a Type-2 CD4 T-cell differentiation [18], which is associated with protumor immunity. Thus, the elevation of FLT3L and CCL24 may 


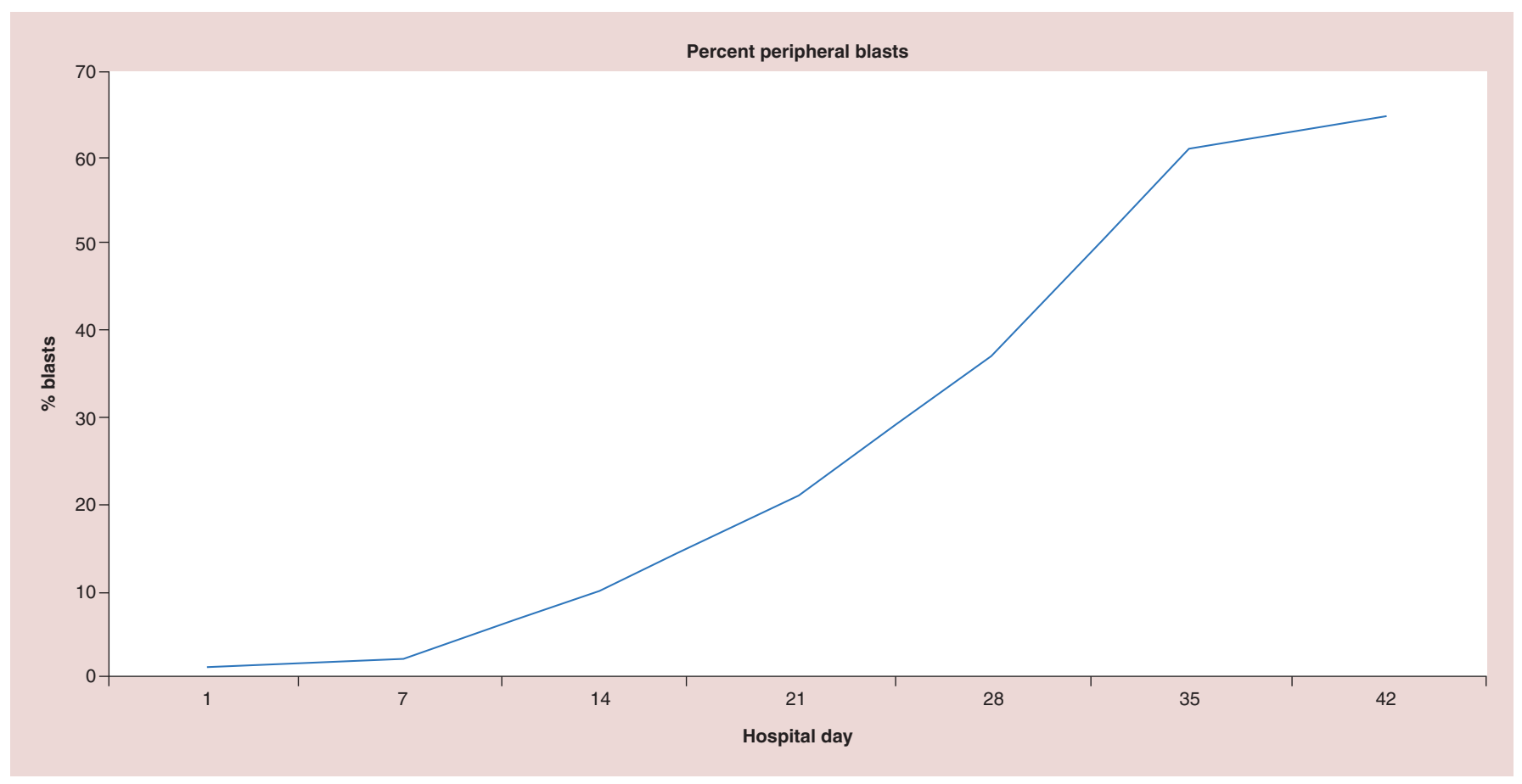

Figure 2. Percent peripheral blasts. The percent of peripheral blasts present during the 6 weeks of epigenetic and checkpoint inhibitor therapy are reported over time (days of therapy).

also be reflective of pro-AML growth and progressive disease. Although a baseline (prenivolumab treatment) serum sample was unavailable for comparison, it is possible that the elevation of these cytokines is a reflection of both activated innate immunity [14] against the AML as well as ongoing AML proliferation, although this is difficult to determine given the lack of pediatric controls.

In summary, this case demonstrates feasibility of combining 5-azacytidine with a PD-L1 and CTLA-4 antibody in a child with refractory leukemia. There were no AEs associated with this therapy over the 6 weeks of treatment and the treatment allowed for improved symptom control at home, limiting the patient's hospital visits and allowed for an improved quality of life. While the cytokine profile is interesting, the anti-AML efficacy of combined epigenetic and checkpoint blockade cannot be determined from this case. Further research is needed to determine if this strategy can be successful for treating pediatric hematologic malignancies such as AML.

Financial \& competing interests disclosure

This publication was supported by the National Center for Advancing Translational Sciences, National Institutes of Health, through Grant Numbers UL1TR001436 and 1TL1TR001437 to L Broglie. Its contents are solely the responsibility of the authors and do not necessarily represent the official views of the $\mathrm{NIH}$. The authors have no other relevant affiliations or financial involvement with any organization or entity with a financial interest in or financial conflict with the subject matter or materials discussed in the manuscript apart from those disclosed.

No writing assistance was utilized in the production of this manuscript.

Ethical conduct of research and informed consent disclosure

The authors state that they have obtained appropriate institutional review board approval or have followed the principles outlined in the Declaration of Helsinki for all human or animal experimental investigations. In addition, for investigations involving human subjects, informed consent has been obtained from the participants involved.

The authors state that they have obtained verbal and written informed consent from the patient/patients for the inclusion of their medical and treatment history within this case report. 
Open access

This work is licensed under the Attribution-NonCommercial-NoDerivatives 4.0 Unported License. To view a copy of this license, visit http://creativecommons.org/licenses/by-nc-nd/4.0/

\section{References}

Papers of special note have been highlighted as: $\bullet$ of interest; $\bullet \bullet$ of considerable interest

1. Gorman MF, Ji L, Ko RH et al. Outcome for children treated for relapsed or refractory acute myelogenous leukemia (rAML): a therapeutic advances in childhood leukemia (TACL) consortium study. Pediatr. Blood Cancer 55(3), 421-429 (2010).

2. Ko RH, Ji L, Barnette P et al. Outcome of patients treated for relapsed or refractory acute lymphoblastic leukemia: a therapeutic advances in childhood leukemia consortium study. J. Clin. Oncol. 28(4), 648-654 (2010).

3. Armand P. Immune checkpoint blockade in hematologic malignancies. Blood 125(22), 3393-3400 (2015).

4. Prieto PA, Yang JC, Sherry RM et al. CTLA-4 blockade with ipilimumab: long-term follow-up of 177 patients with metastatic melanoma. Clin. Cancer Res. 18(7), 2039-2047 (2012).

5. Ansell SM, Lesokhin AM, Borrello I et al. PD-1 blockade with nivolumab in relapsed or refractory Hodgkin's lymphoma. N. Engl. J. Med. 372(4), 311-319 (2015).

- Nivolumab for treatment of relapsed or refractory Hodgkin's lymphoma was able to induce a response rate of $87 \%$, with $17 \%$ of patients achieving a complete response.

6. Berman DM, Wolchok J, Weber J, Hamid O, O’Day S, Chasalow SD. Association of peripheral blood absolute lymphocyte count (ALC) and clinical activity in patients (pts) with advanced melanoma treated with ipilimumab. J. Clin. Oncol. 27(15), 3020 (2009).

7. Esendagli G. A co-stimulatory trap set by myeloid leukemia cells. Oncoimmunology 2(6), e24524 (2013).

-• Myeloid leukemia cells were shown to evade immune response by upregulation of PD-L1.

8. Laurent S, Palmisano GL, Martelli AM et al. CTLA-4 expressed by chemoresistant, as well as untreated, myeloid leukaemia cells can be targeted with ligands to induce apoptosis. Br. J. Haematol. 136(4), 597-608 (2007).

-• CTLA4 is expressed on acute myeloid leukemia blasts in some patients and CTLA4 blockade was able to induce apoptosis of myeloid leukemia cells.

9. Das R, Verma R, Sznol M et al. Combination therapy with anti-CTLA-4 and anti-PD-1 leads to distinct immunologic changes in vivo. J. Immunol. 194(3), 950-959 (2015).

10. Davids MS, Kim HT, Bachireddy P et al. Ipilimumab for patients with relapse after allogeneic transplantation. N. Engl. J. Med. 375(2), 143-153 (2016).

11. Daver N, Basu S, Garcia-Manero G et al. Phase IB/II study of nivolumab in combination with azacytidine (AZA) in patients (pts) with relapsed acute myeloid leukemia (AML). Blood Conference: 58th annual meeting of the American Society of Hematology, ASH 2016. United States; $128: 763$.

-• Nivolumab given in combination with azacytidine was well tolerated in adult patients.

12. Hirahara K, Nakayama T. $\mathrm{CD}_{4}^{+}$T-cell subsets in inflammatory diseases: beyond the Th1/Th2 paradigm. Int. Immunol. 28(4), 163-171 (2016).

13. Dulos J, Carven GJ, van Boxtel SJ et al. PD-1 blockade augments Th1 and Th17 and suppresses Th2 responses in peripheral blood from patients with prostate and advanced melanoma cancer. J. Immunother. 35(2), 169-178 (2012).

- PD-1 inhibition results in a shift to a proinflammatory state with an increase in proinflammatory cytokine expression.

14. Poleganov MA, Bachmann M, Pfeilschifter J, Muhl H. Genome-wide analysis displays marked induction of EBI3/IL-27B in IL-18-activated AML-derived KG1 cells: critical role of two kappaB binding sites in the human EBI3 promotor. Mol. Immunol. 45(10), 2869-2880 (2008).

15. Olsnes AM, Motorin D, Ryningen A, Zaritskey AY, Bruserud O. T lymphocyte chemotactic chemokines in acute myelogenous leukemia (AML): local release by native human AML blasts and systemic levels of CXCL10 (IP-10), CCL5 (RANTES) and CCL17 (TARC). Cancer Immunol. Immunother. 55(7), 830-840 (2006).

16. Sarris AH, Talpaz M, Deisseroth AB, Estrov Z. Human recombinant interferon-inducible protein-10 inhibits the proliferation of normal and acute myelogenous leukemia progenitors. Leukemia 10(5), 757-765 (1996).

17. Dehmel U, Quentmeier H, Drexler HG. Effects of FLT3 ligand on human leukemia cells. II. Agonistic and antgonistic effects of other cytokines. Leukemia 10(2), 271-278 (1996).

18. Miyagaki T, Sugaya M, Fujita $\mathrm{H}$ et al. Eotaxins and CCR3 interaction regulates the Th2 environment of cutaneous T-cell lymphoma. J. Investig. Dermatol. 130(9), 2304-2311 (2010). 